\title{
Cisj
}

\section{DECISION CONCEPTS}

\author{
Miki Sirola \\ Helsinki University of Technology, Laboratory of Computer and Information Science \\ P.O.Box 5400, FIN-02015 HUT, Finland, Miki.Sirola@hut.fi, http://www.cis.hut.fi/miki
}

\begin{abstract}
Decision making is done in many application areas. Still most studies are done in such fields as economy and production planning. In methodologies used there exists more variation. This paper reviews the decision concepts discussed in the literature. Also some decision models by the author are commented. The field and practise in decision science is summarized. Although decision support systems are the final results of many projects, they are mostly based on the decision concepts behind the studies that deserve also more detailed examination. Decision analysis approach and knowledge-based technologies are examples of commonly used concepts.
\end{abstract}

Keywords: Decision making, Decision support systems, Decision support concepts, Knowledge-based systems, System analysis, Modelling

\section{INTRODUCTION}

Good enough decision making is needed in various application areas. The consequences of bad decisions are well known especially in safety critical industrial fields. The responsible decision maker is in most cases a human being or a group of people, but suitable aids to base the decisions on are often welcome.

The problem is to find the proper tools to help the decision making in each case. The tools and methods needed depend on the decision type in question. This paper reviews different decision concepts found in the literature. The decision concepts previously used by the author are also shortly commented. The decision concept field and practise are summarized and discussed.

The literature gives rather large perspective to the whole problem, because this field has been largely studied from various point of views. Still the application areas concentrate on certain areas such as decision making in economical and productional problems. However, the tools and methods used vary on a large scale.

\section{LITERATURE REVIEW}

According to the literature decision sciences concentrate very much on such applications as economy and production planning. Of course also other application areas are involved. The mentioned application areas include decision types that are commonly studied in decision sciences.
The decision types can be divided into long-term decisions and short-term decisions. The number of objectives, possible uncertainty, time dependence, etc. also affects to the perspective how each decision problem should be attached. Sometimes the decisions need to be made on-line and sometimes off-line. All these factors need to be kept in mind in choosing the problem solving methodology.

Some common problem types met within the review are listed next. Decision making in handling of sequences, recourse allocation, network design and sorting problems are examples of in detail studied problem types. Quite common classification problem area leads to such approaches as artificial neural networks.

Decisions can be described as decision trees, decision tables, or flow diagrams. Each of these methodologies has its advantages and disadvantages. Different measures in decision making are interesting points. Deterministic decision making has its own measures mostly based on value (and utility) theory, while stochastic decision making uses statistical measures.

Comparing risk and cost is a common methodology in decision making. Choosing the preferences when the priorities are competing is an important point. Cumulative quality function and chained paired comparisons are examples of more specific methodologies used in decision making, and they are discussed more later on.

According to a survey [1] there are a large number of studies informing us of the design and 
development issues relating to Knowledge-Based Systems (KBS). There seems to be less research examing issues relating to the management and impact of KBS on individuals and organization.

By synthesizing existing research in economics, psychology, and decision sciences and through an ambitious agenda to extend this synthesis into other disciplines, a complete and systematic approach to rational decision making including uncertainties can be reached [2].

Every time managers are faced with a strategic decision they need to decide how to decide. They make choices about who has the necessary information and who needs to take part in the decision. Such responses to strategic issues are believed to be affected by the way in which decision makers interpret issues. Organizations develop habitual responses to issues and may be predisposed because of their attention to rules and routines, or because past performance, to respond to strategic issues in particular ways regardless of how issues are interpreted [3].

Because product development is characterized by time-based competition, there is pressure to make decisions fast using heuristics methods that lead to faster project completion [4]. Pre-emption heuristics are needed both to select activities for pre-emption and then to decide which resources to use to restart pre-empted activities.

Although the expected utility theory has been an expansively accepted normative model in the field of decision science, many researchers still challenge its rationality as "rational decisions making model", because it sometimes fails to comprehensively explain the behaviours of some so-called rational decision makers; such failure has been shown by some paradoxes [5].

The cost functions used to model a variety of manufacturing systems are very often derived from average cost models of unconstrained inventory problems. The use of setups and average inventories as the basis for modelling the economics of a typical batch manufacturing cell is shown to be adequate [6].

The pairwise comparison technique is a building block of the Analytic Hierarchy Process, which has been often used for multi-criteria decision analysis. A shortcut technique has been developed in which only $\mathrm{n}$ paired comparisons forming a closed chain are needed for $\mathrm{n}$ decision elements [7]. Together with the development of a simple and intuitive measure of (in)consistency, this technique of Ra derives the relative weights of decision elements via easy step-by-step calculations on a spreadsheet format.

Information matrices are quite often the output produced by a decision support system. These matrices are an ordinary method for expressing a decision situation under different decision-making scenarios. The decisions involved in designing a decision support system to generate such information matrix are important and involve several costs and benefit components. A designer needs guidance in making effective design decisions in such context. Such guidance is provided by considering the relationships among specific design decisions, costs and benefits [8].

A real application of a multi-criteria decision aid approach to portfolio selection based on preference selection, using ordinal regression and linear programming, has been constructed [9]. The additive utility functions that are derived through this approach have the extrapolation ability that any new alternative can be evaluated and classified into one of several user-predefined groups. The comparison with multiple discriminant analysis - either using a stepwise procedure or not - illustrates the superiority of the mentioned methodology over well-known multivariate statistical technique that has been largely used to study financial decision-making problems.

Logical inference is an important methodology in information and decision sciences. Simulation provides an effective pragmatic approach to detailed analysis and evaluation of supply chain design and management alternatives. Two major decision support methodologies can be considered to be binary logic and a kind of mixed-integer programming model. Stochastic dominance criteria, a rigorous statistical procedure, different learning techniques, data distribution characteristics, and discrimination methods should be mentioned as well in the context of methodological discussion.

A promise of autonomous software agents can be seen [10]. Many benefits of agents and particular characteristics of agents leading to Decision Support System (DSS) enrichment can be noted. A general framework for agent-enabled DSS is concluded to be more difficult to build than traditional DSS, but still some of them bring significant benefits to users. The two well-known challenges in software quality requirements specification and analysis should be kept in mind: software requirements are usually imprecise, and software quality requirements often conflict with each other.

The potential of neural networks for classification problems has been shown by numerous successful applications reported in the literature. The issue of unequal misclassification costs in neural networks classifiers has been investigated in a study [11]. Through an application in theories disease diagnosis, it is found that suitable use of cost information can help in optimal decision making. 
Identifying manufacturers' competitive priorities is considered a key element in manufacturing strategy research. Relatively little effort has been devoted to measurement of these constructs in published research. A study develops scales for commonly accepted competitive priorities, cost importance, quality importance, delivery-time importance, and flexibility importance [12].

Research has indicated the importance of matching information technology applications or manufacturing systems with competitive strategy of a company. The Intelligent Decision Support System (IDSS) consists of an interactive user interface, a knowledge database, a decision model, and a knowledge-based system, as described in various applications. The disciplines of operations and information systems have come closer together as information and process-technology-based changes force manufacturing companies to become more efficient and customer focused.

Geographic Information Systems (GIS) have taken on an increasingly important role supporting decision making in many organizations [13]. GIS have been used to support numerous tasks including oil and mineral exploration, facility location, logistics support, and facilities management decisions. The effect of GIS as a decision support tool comes mostly from the visual display of data in the form of maps. When presenting information as a geographic map, the level of data aggregation possibly affects aspects of task complexity such as information load and the potential for pattern recognition by the user.

The US department of energy has met with the critical and complex decision of choosing technologies for waste site remediation [14]. The focus is to develop a decision support tool that assists the decision maker to find an optimal portfolio. A portfolio consists of selecting the appropriate processes for a remediation site, and choosing a technology for every process so that the decision maker's objectives are achieved. The measures supporting the objectives are risk, lifecycle cost, and time required for remediation. The model uses exponential attribute utility functions with an additive objective function, and provides the decision maker with estimates of the cost and time distributions, and their associated utility. Cumulative frequency distributions illustrate the dominance of technology choices and the variance in the result.

Interruptions are a usual aspect of the work environment in most organizations. Still quite little is known how interruptions and their characteristics (such as frequency of occurrence) influence decision-making performance of individuals. Interruptions seem to improve decision-making performance on simple tasks and to lower performance on complex tasks [15].

The effect of training to reduce biases and heuristics on the consequences of judgements is an important aspect. Untrained judgements may systematically yield better consequences than subjects trained to reduce biases and heuristics.

The ethical point of view has not been thought about so often in the context of decision sciences. Still some examples of this can be found from the literature. For instance, healthcare providers can think ethically about making decisions in many ways including checklists, questions, principles, decision science methods, and consequences [16].

\section{DECISION CONCEPTS USED BY THE AUTHOR}

The author has used several decision concepts in different contexts [17]. In a Computerized Accident Management Support system (CAMS) was built a knowledge-based decision element called strategy generator. This idea was later further developed and a prototype of a rule-based decision model was made. In a very simplified form this idea was also applied in an agent control example. These decision elements were all based on rule-based inference methods combined with some optimization algorithms and traditional calculation techniques.

In a maintenance tool called computerized decision analysis tool (Decision Tool in short form) decision analysis methodology based on value and utility theory was used [17]. This tool and the methodology developed - including a large criteria database for various applications - was tested also in a real decision case by a regulatory body, which was analyzed afterwards.

The author has also tried to combine knowledgebased techniques with artificial neural networks [18]. Decision methodologies used in the Finnish industry has been reviewed as well [19]. In reference [20] knowledge-based techniques have been combined with fuzzy logic and some other traditional techniques in a decision model that has been built for state monitoring purposes. In reference [21] an elder case previously handled basically by knowledge-based technologies (described in detail in reference [17]) has been analyzed with decision analysis methodology including comparisons to the elder case. Also a new decision model based on a combination of several known decision concepts is under construction. This model will be described in detail later in another paper.

More technical details about the decision support systems composed by the author can be found from references [17-21]. Many of these techniques have 
also been tested in practise. More information about this is also written in the mentioned references. The author has quite an experience from various decision support systems constructed during the last decade.

\section{CONCLUSION}

Different decision concepts found in the literature have been reviewed. Decision concepts are needed in very many fields, but the research seems to concentrate on such applications as decision making in economy and production planning. From methodological point of view the scale is much larger.

Decisions can be described and measured in different ways. Also decision types have variations, and therefore different aids suit to different problems in decision support. Risk and cost are often compared, and preferences between different priorities need to be checked.

Knowledge-based systems have already for a long time been used in dealing with various decision making issues. Autonomous software agents and neural networks are also promising methods in this area. Logical inference with help of some simulation techniques can be considered one of the very basic methodologies used.

Decision analysis methodology is one basic methodology that has been widely studied. This method consists of very many smaller parts that cannot all be discussed here in detail. Many of these parts constitute research fields of their own. Chained paired comparisons [7] is an example of such a more specific methodology developed, and it is also discussed in this paper.

More technical details and ways of solving various tasks including examples can be found from the references. Both author self and other referred authors have been able to explain through examples how a certain methodology works in each problem case analysed.

A promising decision support system requires a functioning decision concept behind. Without a reasonable concept the support system cannot fulfil all its requirements. Therefore the studies of decision concepts itself can be regarded as even more important than the decision support systems itself. Each practise needs a theory or at least a competent and effective methodology behind of it. Of course the final decision support system can be regarded as a fruit of all this work.

\section{REFERENCES}

[1] R. Santhanam, J. Elam. A survey of knowledge-based systems research in decision sciences (1980-1995). The Journal of the Operational Research Society. Oxford. (1998).
[2] A. Lo. The three P's of total risk management. Financial Analysts Journal. Charlottesville. (1999).

[3] D. Ashmos, D. Duchon and R. McDaniel. Participation in strategic decision making: the role of organizational predispositions and issue interpretation. Decision sciences. Atlanta. (1998).

[4] R. Ash, D. Smith-Daniels. The effects of learning on decision rule performance in multiproject scheduling. Decision sciences. Atlanta. (1999).

[5] C. Sheng, H. Lin. Revised utility function, allias' choice, and their application to investment decision making. International Journal of Management. Poole. (1999).

[6] J. Rummel. An empirical investigation of costs in batching decisions. Decision sciences. Atlanta. (2000).

[7] J. Ra. Chainwise paired comparisons. Decision sciences. Atlanta. (1999).

[8] A. Karim, J. Hershauer and W. Perkins. A simulation of partial information use in decision making: implications for DSS design. Decision sciences. Atlanta. (1998).

[9] C. Zopounidis, M. Doumpos. Stock evaluation using preference disaggregation methodology. Decision sciences. Atlanta. (1999).

[10] T. Hess, L. Rees and T. Rakes. Using autonomous software agents to create next generation of decision support systems. Decision sciences. Atlanta. (2000)

[11] V. Berardi, G. Zhang. The effect of misclassification costs on neural network classifiers. Decision sciences. Atlanta. (1999).

[12] P. Ward, J. McCeery, L. Ritzman and D. Sharma. Competitive priorities in operations management. Decision sciences. Atlanta. (1998).

[13] M. Swink, C. Speier. Presenting geographic information: effects of data aggregation, dispersion, and users' spatial orientation. Decision sciences. Atlanta. (1999).

[14] J. Jackson, J. Kloeber and B. Ralston. Selecting a portfolio of technologies: an application of decision analysis. Decision sciences. Atlanta. (1999).

[15] C. Speier, J. Valacich and I. Vessey. The influence of task interruption on individual decision making: an information overload perspective. Decision sciences. Atlanta. (1999).

[16] J. Worthley. The ethical dimension of ordinary professional life. Healthcare Executive. Chicago. (1999).

[17] M. Sirola. Computerized decision support systems in failure and maintenance management of safety critical processes. VTT Publications 397. Espoo, Finland, 1999. 
[18] M. Sirola, J. Vesanto. Utilization of neural methods in knowledge-based decision support systems - state monitoring as a case example. Proceedings of IASTED International Conference in Modelling, Identification and Control. Innsbruck, Austria, 2000.

[19] M. Sirola. Methodologies used in computerized decision support systems of safety critical processes - comparison to Finnish industry. Proceedings of IASTED International Conference in Modelling, Identification and Control. Innsbruck, Austria, 2000.

[20] M. Sirola. Decision model for state monitoring. Proceedings of IASTED International Conference in Modelling, Identification and Control. Innsbruck, Austria, 2001.

[21] M. Sirola. Applying decision analysis method in process control problem in accident management situation. Proceedings of XIV International Conference on System Science. Wroclaw, Poland, 2001.

\footnotetext{
Miki Sirola has been a Laboratory Engineer in the Laboratory of Computer and Information Science in Helsinki University of Technology since 1998. He received MSc 1988 in Electrical Engineering (System Control and Automation) from

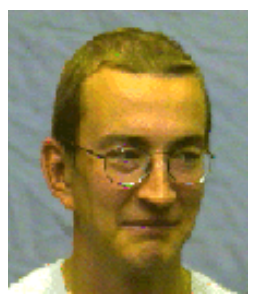

Helsinki University of Technology, LicTech 1993 in Electrical Engineering (Automation) and DTech 1999 in Automation and System Technology (Automation) from the same university. Prior to Helsinki University of Technology, he worked at VTT (Technical Research Centre of Finland) Automation as research scientist (1987-1998) and at Institutt for Energiteknikk (OECD Halden Reactor Project) as research scientist (1992-1993). His research interests are in knowledge-based systems and decision support.
} 